4. Epidemiologisches Krebsregister Niedersachsen (Hrsg) (2004) Krebs in Niedersachsen. Registerstelle des Epidemiologischen Krebsregisters Niedersachsen, Oldenburg

5. Gonzalez CA, Riboli E (2010) Diet and cancer: contributions from the European prospective investigation into cancer and nutrition (EPIC) study. Eur J Cancer 46(14):2555-2562

6. Gordis L (2008) Epidemiologie. Kilian, Postbauer-Heng

7. Key TJ, Allen NE, Spencer EA et al (2002) The effect of diet on risk of cancer. Lancet 360:861-868

8. Kreienbrock L, Schach S (1995) Epidemiologische Methoden. Fischer, Jena

9. Norat T, Bingham S, Ferrari P et al (2005) Meat, fish, and colorectal cancer risk: the European prospective investigation into cancer and nutrition. J Natl Cancer Inst 97(12):906-916

10. RKI (Robert-Koch-Institut), Gesellschaft der epidemiologischen Krebsregister in Deutschland e.V. (2010) Krebs in Deutschland 2005/2006 - Häufigkeiten und Trends. Gesundheitsberichterstattung des Bundes, Statisches Bundesamt, Zweigstelle Bonn

11. Rothman KJ, Greenland S, Lash T (2008) Modern epidemiology. Kluwer Lippincott Williams \& Wilkins, Philadelphia

12. Steindorf K, Jedrychowski W, Schmidt M et al (2005) Casecontrol study of lifetime occupational and recreational physical activity and risks of colon and rectal cancer. Eur J Cancer Prev 14(4):363-371

13. Wahrendorf J (1991) Grundbegriffe der Epidemiologie. In: Gundermann KJ, Rüden H, Sonntag HG (eds) Lehrbuch der Hygiene. Fischer, Stuttgart, S 553-570, 616-617

\section{Schützt Grüner Tee vor Darmkrebs?}

\section{Kann Grüner Tee Darmkrebs vorbeugen? Dieser Frage gehen Wissenschaftler der Universitäten in Halle/Saale und in Ulm nun in einer Langzeitstudie nach. Die Deutsche Krebshilfe fördert das weltweit größte Forschungsvorhaben zu dieser Fragestellung mit insgesamt 2,1 Millionen Euro.}

Grüner Tee gehört zu den ältesten und am weitesten verbreiteten Getränken der Welt. Er wird hauptsächlich aus den Blättern der Pflanze Camellia sinensis hergestellt. Bereits vor einigen Jahren haben Forscher herausgefunden, dass ein bestimmter Inhaltsstoff des Grünen Tees, das Pflanzenhormon Epigallocatechin-3gallat (ECGC), vor bestimmten Krebsarten schützen kann. In verschiedenen Forschungsarbeiten haben sie beobachtet, dass ECGC die Entstehung von Tumoren der Prostata, Brust und Gebärmutter hemmt, heißt es in der Mitteilung der Deutschen Krebshilfe.

\section{Tägliche Dosis Grüntee-Extrakt}

Das Forschungsteam von der Klinik und Poliklinik für Innere Medizin I des Universitätsklinikums Halle und vom Institut für Naturheilkunde und Klinische Pharmakologie der Universität Ulm untersuchen nun den Nutzen von Grünem Tee zur Darmkrebsprävention. Zielgruppe der Studie sind Patienten, bei denen bei einer Koloskopie im Rahmen der Krebsfrüherkennung bereits Polypen entdeckt und entfernt wurden. Ob bei diesen Patienten durch den regelmäßigen Genuss von Grünem Tee vermieden werden kann, dass sich neue Polypen bilden, wollen die Wissenschaftler nun herausfinden. Dazu verabreichen sie jedem Versuchsteilnehmer eine tägliche Dosis Grüntee-Extrakt in Form von Kapseln. Diese werden über einen Zeitraum von drei Jahren eingenommen. Eine Tagesdosis besteht aus zwei Kapseln, die jeweils $150 \mathrm{mg}$ des Catechins und Antioxidans Epigallocatechingallat (EGCG) enthalten. Zum Vergleich: Eine Tasse Grüner Tee enthält etwa 50 bis $70 \mathrm{mg}$ des Wirkstoffs.

Alle vier Monate entnehmen die Ärzte den Patienten Blut, um ihre Leber- und Blutwerte zu kontrollieren. Außerdem fragen sie die Studienteilnehmer regelmäßig, ob diese die Kapseln auch eingenommen, zusätzlich Grünen Tee getrunken oder andere Medikamente bekommen haben. Zudem wird nach drei Jahren mit einer Koloskopie ermittelt, ob und wie viele neue Polypen sich gebildet haben.

\section{Studien:}

- Bettuzzi S, Brausi M, Rizzi F et al. (2005) Chemoprevention of Human Prostate Cancer by Oral Administration of Green Tea Catechins in Volunteers with High-Grade Prostate Intraepithelial Neoplasia: A Preliminary Report from a One-Year Proof-of-Principle Study. Association for Cancer Research

- Seely D, Mills EJ, Wu P et al. (2005) The Effects of Green Tea Consumption on Incidence of Breast Cancer and Recurrence of Breast Cancer: A Systematic Review and Meta-analysis. Integr Cancer Ther.

- Ahn WS, Yoo J, Huh SW et al. (2003) Protective effects of green tea extracts (polyphenon $E$ and EGCG) on human cervical lesions. European Journal of Cancer Prevention.

Quelle: Ärzte Zeitung, www.aerztezeitung.de 